\title{
Sozialräumliche Monitoringsysteme. Ein Vergleich quantitativer Herangehensweisen für städtische Sozialraummonitorings im deutschsprachigen Raum
}

\author{
Markus Speringer $\mathbb{0}$, Mira Böing \\ Eingegangen: 19. Februar 2021 - Angenommen: 16. September 2021 - Online veröffentlicht: 14. Oktober 2021
}

\section{Zusammenfassung}

Städte sind Zentren der sozialen, kulturellen, wirtschaftlichen und materiellen Vielfalt, in denen Menschen in unterschiedlichsten Lebenslagen im dicht besiedelten Raum aufeinandertreffen und sich soziale Ungleichheiten bzw. Disparitäten durch die räumliche Konzentration von Bevölkerungsgruppen manifestieren und verstärken können. Durch die Zunahme innerstädtischer Disparitäten haben zahlreiche deutschsprachige (Groß-)Städte begonnen, kleinräumige Sozialraumberichterstattungen aufzubauen, um der räumlichen Konzentration von sozialen Problemlagen frühzeitig entgegenwirken zu können. In diesem Beitrag werden die unterschiedlichen methodisch-konzeptionellen Herangehensweisen bei quantitativen Sozialraumanalysen in 25 deutschsprachigen (Groß-)Städten mit vergleichbarem Datenmaterial systematisch analysiert und diskutiert. Dabei sind für diese Metaanalyse die systematische Gegenüberstellung der Motivationen, Ziele und inhaltlichen Fragestellungen sowie die dafür verwendeten Indikatoren, räumlichen Analyseebenen und methodischen Herangehensweisen von Interesse. Der Erkenntnisgewinn kann für Stadt- und Regionalverwaltungen von Relevanz bei der (Neu-)Konzeption und Durchführung eigener Sozialraumanalysen sein.

Markus Speringer, Magistratsabteilung für Wirtschaft, Arbeit und Statistik (bis 08/2020 Magistratsabteilung für Stadtentwicklung und Stadtplanung), Stadt Wien, Meiereistraße 7, 1020 Wien, Österreich

markus.speringer@wien.gv.at

Mira Böing, Universität Siegen, Hölderlinstraße 3, 57076 Siegen, Deutschland

mira.boeing@uni-siegen.de

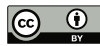

(c) 2021 Speringer; licensee oekom verlag. This Open Access article is published under a Creative Commons Attribution 4.0 International License.
Schlüsselwörter: Sozialraumanalyse Sozialraummonitoring - Stadtforschung - Quantitative Sozialforschung

\section{Social area Monitoring Systems. A Comparison of Quantitative Approaches in Urban Social Aea Monitorings in German Speaking Countries}

\begin{abstract}
Cities are centres of social, cultural, economic and material diversity, where people in the most diverse living situations meet in densely populated areas and where social inequalities or disparities can manifest and intensify through the spatial concentration of population groups. Due to the increase in inner-city disparities, numerous German-speaking (large) cities have started to establish small-scale social area reports in order to be able to counteract the spatial concentration of social problems at an early stage. This paper systematically analyses and discusses the different methodological-conceptual approaches to quantitative social area analyses in 25 Germanspeaking (large) cities with comparable data material. The systematic comparison of motivations, goals and content-related questions as well as the indicators, spatial levels of analysis and methodological approaches used are of interest for this meta-analysis. The knowledge gained can be of relevance for urban and regional administrations when (re)designing and implementing their own social area analyses.
\end{abstract}

Keywords: Social area analysis - Social monitoring - Urban research - Quantitative social research 


\section{Einleitung}

Städte sind Zentren der sozialen, kulturellen, wirtschaftlichen und materiellen Vielfalt, in denen Menschen in unterschiedlichsten Lebenslagen im dicht besiedelten Raum aufeinandertreffen und sich soziale Ungleichheiten bzw. Disparitäten durch die räumliche Konzentration von Bevölkerungsgruppen manifestieren und verstärken können. Dabei werden soziale Disparitäten an sozioökonomischen Kriterien, wie z. B. Bildungsstand, Beschäftigung, Einkommen und materiellem Wohlstand, Wohn- und Eigentumsverhältnissen festgemacht (Lawrence 2002: 35).

Die Zunahme sozialer Disparitäten wird oftmals mit einer sich verändernden Bevölkerungszusammensetzung, den sich wandelnden lokalen, nationalen und internationalen Wirtschaftssystemen (Globalisierung) sowie der Zunahme internationaler Zuwanderung, kultureller Vielfalt, neuen Familien- und Haushaltsformen, höherem Wettbewerb am Arbeitsmarkt, der Zunahme prekärer Arbeitsverhältnisse bei unsicherem Einkommen oder steigenden Einkommensunterschieden assoziiert (Mau 2018: 49). Die sich gleichwohl verstärkende Kluft zwischen Arm und Reich lässt sich auf unterschiedlichen räumlichen bzw. sozialen Ebenen feststellen, welche von der Kontinental- bzw. Länderebene (Milanović 2016: 127) bis auf die individuelle Ebene (Keeley 2015: 66) zwischen Bewohnerinnen und Bewohnern der gleichen administrativen Einheiten (z. B. Stadtteile) oder innerhalb sozialer Gruppen reicht (Schulte-Haller 2011: 5). Dies kann zur sozialen Exklusion gesellschaftlicher Gruppen - unter anderem durch fehlenden Zugang zu öffentlichen Einrichtungen (z. B. Kultureinrichtungen), fehlende Finanzmittel zur Essenversorgung (z. B. Restaurantbesuche, Einkauf im Supermarkt), fehlendem Zugang zu leistbarem Wohnraum - führen, welche sich räumlich in einer residentiellen Segregation von Bevölkerungsteilen manifestieren kann (Lawrence 2002: 34; Glaeser/Resseger/Tobio 2009: 643; OECD 2018: 19; Nijman/Wei 2020: 5).

Eine Beobachtung zunehmender innerstädtischer sozialer Disparitäten hat zahlreiche deutschsprachige (Groß-) Städte veranlasst, kleinräumige quantitative Sozialraumberichterstattungen aufzubauen, um evidenzbasiert der räumlichen Konzentration von sozialen Problemlagen frühzeitig entgegenwirken zu können (Mardorf 2006: 23; Hartwig 2009: 9; Dohnke 2012: 144; Henckel/Kramer 2019: 9; Pohl/Ott 2019: 283). Diese sogenannten Sozialraumanalysen (englisch: Social Area Analysis - SRA) sollen politische Handlungsbedarfe identifizieren und planerische Maßnahmen anleiten. Ihre konzeptionelle Grundlage haben die Sozialraumanalysen in der Denktradition der humanökologischen Chicagoer Schule der 1920er-Jahre, wobei deren methodische Umlegung auf die Social Area Analysis von Shevky und Bell (1961) zurückgeht (Pohl/Ott 2019: 285).
Die (quantitative) Sozialraumanalyse setzt sich mit den demographischen, sozioökonomischen und soziokulturellen Dimensionen sowie baulichen Merkmalen der innerstädtischen Strukturen und Veränderungsprozesse auseinander (Heye/Leuthold 2004: 3).

Seit ihren Ursprüngen hat sich die Sozialraumanalyse von einer Methode der explorativen Stadtforschung zu einem Instrument der angewandten Sozialraumberichterstattung in der Stadtplanung entwickelt, wo häufig von Sozialraummonitoring die Rede ist. ${ }^{1}$ Die geänderte Terminologie zu ,Monitoring' weist bereits auf die Regelmäßigkeit solcher Analysen hin, welche im Gegensatz zu ,klassischen' Sozialraumanalysen (Querschnittsanalysen) kontinuierlich in regelmäßigen Abständen durchgeführt werden (Henckel/Kramer 2019: 10; Pohl/Ott 2019: 283).

Allerdings sind urbane Räume bei Weitem nicht der einzige Untersuchungsgegenstand sozialräumlicher Analysen. So werden auch ländliche Räume (vgl. für Deutschland Küpper/Peters 2019; für Niedersachsen Küpper/Steinführer 2017) oder andere Gebietskörperschaften, wie Regionen (vgl. für die Region Pinzgau (Österreich) Heintel/Speringer/ Schnelzer et al. 2017; für die Region Südliches Waldviertel-Nibelungengau (Österreich) Speringer/Schnelzer/ Heintel 2020) oder Bundesländer (vgl. für Niedersachsen Wunderlich 2019) hinsichtlich ihrer demographischen und sozioökonomischen Strukturen analysiert.

Indes gibt es in der sozialwissenschaftlichen Forschung eine Reihe von Veröffentlichungen, welche Konzeptionen und Methoden von Sozialraumberichterstattungen anhand einzelner Fallbeispiele untersuchen und reflektieren (Hartwig 2009: 27; Pohl/Ott 2019: 289). Komparative Gegenüberstellungen sind hingegen selten oder veraltet (Dohnke 2012: 144). ${ }^{2}$

In diesem Beitrag wird versucht, diese vermeintliche Forschungslücke zu schließen, indem mittels einer Metaanalyse die unterschiedlichen konzeptionellen und methodischen Herangehensweisen bei quantitativen Sozialraumanalysen in 25 deutschsprachigen (Groß-)Städten mit vergleichbarem Datenmaterial systematisch analysiert und diskutiert werden. Die Fokussierung auf urbane Untersuchungsregionen im deutschsprachigen Raum erfolgte vorrangig aus pragma-

\footnotetext{
${ }^{1}$ Die Begriffe Sozialraumanalyse und Sozialraummonitoring werden im Folgenden synonym für quantitative Sozialraumberichterstattungen verwendet. Qualitativ ausgerichtete Sozialraumanalysen wurden dementsprechend nicht erfasst.

2 Durchgeführt vom Deutschen Institut für Urbanistik (Difu) sowie Hartmut Häußermann (res urbana). Betrachtet wurden die Monitoringsysteme der Städte Berlin, Bremen, Hamburg, Mainz, München, Leipzig und Karlsruhe. Dabei wurde zwischen problemorientierten und umfassenden Ansätzen in Sozialraumanalysen unterschieden (Dohnke 2012: 145).
} 
tischen Gründen, da Großstädte vergleichbare demographische und sozioökonomische Strukturen, Prozesse und Sozialräume aufweisen, wo sich stadtspezifische soziale, ökonomische und demographische Problemlagen überlagern und ähnliche sozialpolitische Handlungsbedarfe manifestieren können. Damit vereint Großstädte, dass sie in den Sozialraumanalysen ähnlichen Fragestellungen nachgehen, womit sie ein geeignetes Untersuchungsobjekt für diese Analyse bilden.

Die Studie ist aus der Motivation heraus entstanden, inhaltliche und methodische Rückschlüsse für die (Neu-)Konzeption einer Wiener Sozialraumanalyse ziehen zu können, um die Verwertbarkeit einer solchen Sozialraumanalyse für stadtplanerische und sozialpolitische Belange zu optimieren. Der Erkenntnisgewinn kann auch für andere Städte und Regionen von Relevanz bei der (Neu-)Konzeption und Durchführung eigener Sozialraumanalysen sein.

Unser Ziel ist es, die Kernelemente in der Konzeptionierung und Operationalisierung von Sozialraumanalysen zu identifizieren und mittels systematischer Gegenüberstellung in ein quantitativ auswertbares Analyseraster zu bringen. So lassen sich vergleichende Aussagen über die unterschiedlichen Herangehensweisen treffen und etwaige Handlungsempfehlungen zur Durchführung einer kleinräumigen quantitativen Sozialraumanalyse formulieren.

Zentral sind die folgenden Fragestellungen: (A) Welche Zielsetzung und Motivation werden verfolgt? (B) Welche Methodik wird herangezogen? (C) Welche Raumeinheiten werden analysiert? (D) Welche Merkmale bzw. Indikatoren werden zur Darstellung der räumlichen Sozialstruktur genutzt? (E) Wie erfolgt die Operationalisierung der Merkmale in Indikatoren? (F) Wie häufig werden die Merkmale genutzt? (G) Welche Merkmale treten häufig in Kombination miteinander auf?

Im folgenden Kapitel 2 werden im Rahmen einer theoretischen Einführung die Kernannahmen der städtischen Segregationsforschung thematisiert. Dabei werden zentrale Konzepte sozialer Ungleichheit (Kapitel 2.1), welche das theoretische Grundgerüst dieser Analyse ausmachen, konzeptionelle Herausforderungen von Sozialraumanalysen (Kapitel 2.2) sowie verschiedene Möglichkeiten der methodischen Vogehensweise (Kapitel 2.3) vorgestellt. Im Anschluss erfolgt eine Darstellung der Methodik dieser Analyse (Kapitel 3) sowie der Auswertungsergebnisse (Kapitel 4). Zum Abschluss wird in Kapitel 5 eine kritische Reflexion der beschriebenen Ergebnisse sowie eine Einschätzung zur Optimierung städtischer Sozialraumberichterstattungen durchgeführt.

\section{Die Stadt als Sozialraum}

Ähnlich wie Städte einen Funktionsraum darstellen, in dem sich verschiedene Nutzungen ungleichmäßig über das Stadtgebiet verteilen, bildet die Bevölkerung parallel dazu einen Sozialraum $^{3}$, der vor allem durch eine ungleiche räumliche Verteilung der Wohnstandorte verschiedener sozialer Bevölkerungsgruppen geprägt ist (Farwick 2012: 381). Die sozialräumliche Struktur einer Stadt ist das Ergebnis komplexer Prozesse, in deren Verlauf die unterschiedlichen sozialen Gruppen und Milieus ihren Ort in der Stadt finden bzw. zugewiesen bekommen. Gleichzeitig muss die sozialräumliche Struktur einer Stadt im Kontext des jeweiligen historischen und vorherrschenden ökonomischen, politischen und gesellschaftlichen Systems betrachtet werden (Häußermann/Siebel 2004: 153).

Die sichtbar werdende Verortung sozialer Ungleichheit im Raum steht im Widerspruch zu den normativen Idealvorstellungen einer „Europäischen Stadt“ als Ort der sozialen Integration, der individuellen Freiheit, gemischter Stadtquartiere und gelebter Nachbarschaften (Schmidt 2011: 231; Siebel 2012: 204).

Soziale Segregation beschreibt in der soziologischen Stadtforschung die ungleichmäßige Konzentration von verschiedenen sozialen Gruppen - unter anderem auf Basis demographischer, soziokultureller oder ökonomischer Merkmale - in unterschiedlichen städtischen Teilräumen. Die sozialräumlichen Unterschiede sind beispielsweise an sozioökonomischen Merkmalen wie dem Einkommen, der Berufsqualifikation oder dem Bildungsstand, aber auch ,weichen" Indikatoren wie Werteorientierungen oder Einstellungen ablesbar (Häußermann/Siebel 2004: 143). Der Wohnungsmarkt bildet dabei den entscheidenden Mechanismus der räumlichen Verteilung (Farwick 2012: 383). Während eine soziale Durchmischung unterschiedlicher Bevölkerungsgruppen - positiv konnotiert - häufig als Garant für die Stabilität von Quartieren gilt, werden insbesondere bei einer Konzentration von Armutslagen negative Folgen für die Lebenschancen der Bewohnerinnen und Bewohner sowie baulich-strukturelle Abwertungen im Wohnquartier diskutiert (Glatter/Wiest 2008: 62; Döringer/Uchiyama/ Penker et al. 2020: 1697).

Hingegen muss eine räumliche Konzentration von Bevölkerungsgruppen nach bestimmten Merkmalen keine intendierte Trennung sozialer Gruppen im Stadtgebiet bedeuten und eine soziale Mischung in einem Gebiet muss die soziale

\footnotetext{
3 Der soziale Raum, im Verständnis von Bourdieu (1982), bildet diese sozialen Unterschiede in der Gesellschaft auf der Basis der unterschiedlichen Ausstattungen mit kulturellem und ökonomischem Kapital ab (Heye/Leuthold 2004: 3).
} 
Distanz der unterschiedlichen Bevölkerungsgruppen nicht automatisch aufheben (Häußermann/Siebel 2004: 146). Soziale Ungleichheit innerhalb einer Gesellschaft wird keineswegs durch eine sozialräumliche Mischung unterschiedlicher Bevölkerungsgruppen alleine beseitigt. Stadtpolitisch relevant wird räumliche Segregation auf der Grundlage der Annahme, dass sich soziale Ungleichheit durch die Konzentration benachteiligter Haushalte verfestigen oder verschärfen kann, wie sie in der Kontexteffekteforschung diskutiert wird (Häußermann 2010: 23; Friedrichs 2013: 18). Segregierte Wohngebiete, in denen sich vermehrt Angehörige der oberen Mittelschicht und Oberschicht konzentrieren, sind kaum Gegenstand der Kontexteffekteforschung (Friedrichs 2013: 37). Dabei hat die deutschsprachige Stadtforschung Themen wie die aufkeimende Gentrifizierung von Stadtvierteln schon vor geraumer Zeit für sich entdeckt, um die vielschichtigen dahinterstehenden Prozesse, wie positiv konnotierte Aufwertungsprozesse (z. B. Schaffung oder Instandsetzung von Infrastrukturen, Renovierungstätigkeiten), im Kontrast zu den daraus resultierenden Verdrängungsmechanismen von einkommensschwachen Bevölkerungsgruppen (z. B. Mietpreiserhöhung) zu untersuchen (Dirksmeier 2010; Franz 2015; Helbrecht 2016: 11; Schnelzer 2018; Kadi/Verlič 2019).

Dass Politik und Verwaltung auf soziale Segregationstendenzen in städtischen Räumen reagieren, kann am Beispiel der Stadt Wien aufgezeigt werden. Bereits im 19. Jahrhundert, als die Industrialisierung die europäischen Städte - begleitet von einem rasanten Bevölkerungswachstum vor die Herausforderungen massiver Wohnungsnot und zunehmender Verelendung von Stadtvierteln stellte, wurde in Wien, vor allem zu Beginn des 20. Jahrhunderts, massiv in öffentlich geplante und finanzierte Bautätigkeiten (z. B. Gemeindebau oder Genossenschaften) investiert, welche das Wiener Stadtbild flächendeckend bis heute prägen (Bauer/ Haydn 2019: 38).

Vergleichbare stadtplanerische Entwicklungen zum Ausgleich sozialer Disparitäten im Stadtgebiet durch Förderung des gemeinnützigen Wohnbaus oder Programme sozialer Stadtentwicklung, die darauf zielen, die Lebensbedingungen der Bevölkerung in segregierten Gebieten zu verbessern, sind im gesamten deutschsprachigen Raum zu finden, wie es beispielhaft das in Deutschland von Bund, Ländern und Gemeinden gemeinsam finanzierte Programm „Sozialer Zusammenhalt" zeigt. ${ }^{4}$ Während hier der Fokus auf benachteiligten Stadtteilen liegt, werden zuletzt gehäuft auch auf gesamstädtischer Ebene stadtpolitische Instrumente zur

${ }^{4}$ https://www.staedtebaufoerderung.info/DE/Programme/ SozialerZusammenhalt/sozialerzusammenhalt_node.html (12.08.2021).
Regulierung des Boden- und Wohnungsmarktes diskutiert und angewendet (BBSR 2019: 98; Gennies 2021: 45).

\subsection{Konzeptionen sozialer Ungleichheit}

Bei der Durchführung einer städtischen Sozialraumanalyse stellt sich die Frage, anhand welcher Merkmale die Bevölkerungsstruktur einer Stadt veranschaulicht werden soll. Bei der Indikatorauswahl sollte berücksichtigt werden, dass das, was als Kriterien für die Bestimmung sozialer Ungleichheit einer Gesellschaft relevant gemacht wird, an den jeweiligen historischen Kontext gebunden ist und somit nie vollständig objektiv sein kann (Burzan 2011: 7; Mau 2018: 23). Letztlich muss im Rahmen eines reflexiven Planungsprozesses entschieden werden, welche Kategorien für die Beschreibung von Bevölkerungsgruppen wichtig erscheinen, das heißt, welche Maßzahlen die gesellschaftliche Heterogenität am besten abbilden.

Innerhalb des Theoriespektrums der Sozialstrukturanalyse moderner Gesellschaften werden unterschiedliche Ursachen und Merkmale sozialer Ungleichheit genannt (Burzan 2011: 15). Entsprechend ihrer Analyseebenen können vier zentrale Ansätze aufgeführt werden, namentlich (1) Soziale Schichten und Klassen, (2) Modell der sozialen Lage, (3) Modell der sozialen Milieus und Lebensstile, sowie (4) Modell der Exklusion und Inklusion.

Die in quantitativen Sozialraumanalysen genutzten Analysedimensionen lassen sich am ehesten mit dem Modell der sozialen Lage (auch Lebenslagenkonzept) fassen, da durch die Auswahl der Indikatoren (z. B. Herkunft, Lebensform) implizit darauf Bezug genommen wird. Das Modell der sozialen Lage wurde Ende der 1980er-Jahre mit der Zielsetzung entwickelt, die gesellschaftliche Ungleichheitsstruktur multidimensional zu erfassen (Hradil 1987: 13). Dabei werden neben vertikalen sozioökonomischen Merkmalen auch horizontale wie Geschlecht, Alter, Herkunft, Region oder unterschiedliche Lebensformen berücksichtigt (Geißler 2014: 77). Ziel dieses mehrdimensionalen Ungleichheitsmodells ist es, eine differenzierte Beschreibung objektiv messbarer Lebensbedingungen anzustreben (Burzan 2011: 140). Die unterschiedlichen Lagen müssen indes nicht notwendigerweise, wie in Klassen- und Schichtkonzepten, hierarchisch angeordnet sein. Es können sich aber ebenfalls vorteilhafte und nachteilige Lebensbedingungen aus den spezifischen Lagen relational zueinander ergeben (Burzan 2011: 140). Die Stärke des Modells liegt also im Vergleich zu den anderen Modellen in der detaillierten, quantitativ messbaren und nichthierarchischen Beschreibung empirischer Lebensverhältnisse, was es für die Konzeptionierung von Sozialraumanalysen anschlussfähig macht. 


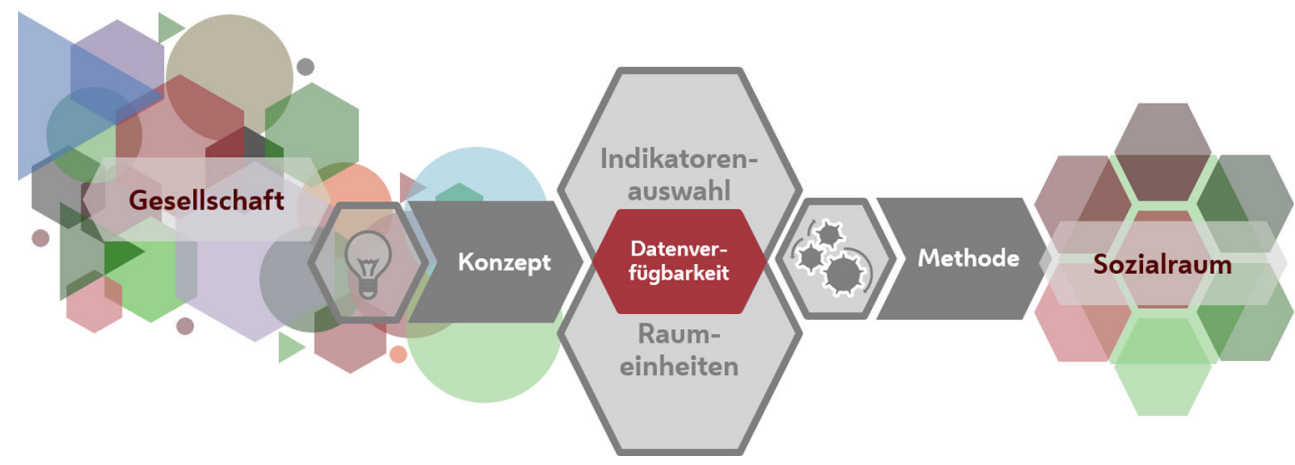

Abbildung 1 Kernelemente einer quantitativen Sozialraumanalyse

\subsection{Kernelemente von Sozialraumanalysen}

Die Quantifizierung komplexer gesellschaftlicher Strukturen und Prozesse mittels Sozialraumanalysen versucht den Sozialraum anhand ausgewählter Kernelemente - namentlich die Motivationen, Ziele und inhaltlichen Fragestellungen sowie die verwendeten Indikatoren, räumlichen Analyseebenen und methodischen Herangehensweisen - in den jeweiligen Sozialraumanalysen abzubilden bzw. diese auf vermeintlich repräsentative Merkmale zu reduzieren. Diese Dimensionsreduktion führt unweigerlich zu einem Informationsverlust bzw. zu einer Generalisierung sozialräumlicher Strukturen, welche blinde Flecken in der Analyse produzieren kann. Aus diesem Grund bedarf es vor der Durchführung einer Sozialraumanalyse umfangreicher konzeptioneller Vorüberlegungen, um den Analysefokus nicht zu verlieren.

Abbildung 1 ist ein Versuch, den hinter einer Sozialraumanalyse steckenden Prozess schematisch aufzubereiten. Den Ausgangspunkt zur quantitativen Abbildung der Gesellschaft bzw. des ,realen ' Sozialraums mittels Sozialraumanalyse bildet die Reflexion über die stadtplanerische Motivation bzw. Zielsetzung (dargestellt als Glühbirne) hinter einer solchen Analyse. Dementsprechend muss man sich eingangs im Klaren sein, welche Fragestellung es zu beantworten gilt bzw. was das zentrale Untersuchungsobjekt (z. B. Benachteiligung, Armut) ist. Das beeinflusst im Planungsprozess der jeweiligen Sozialraumanalyse sowohl den thematischen Fokus, die Auswahl der Indikatoren, die zu wählenden Raumeinheiten als auch letztlich die im Sinne der Zielsetzung passende methodische Herangehensweise. Einschränkend wirkt hier der Faktor Datenverfügbarkeit bzw. Datenzugang. Diese Elemente fungieren als Eckpfeiler jeder sozialräumlichen Analyse, weshalb diese für die vorliegende komparative Gegenüberstellung unterschiedlicher Herangehensweisen in der Stadtplanung als Analysedimensionen genutzt werden.

Generell soll die Sozialraumberichterstattung, als Teilbereich der Sozialstatistik, den Zustand sowie die Verände- rungen der Lebensbedingungen, wie z. B. den materiellen Lebensstandard, der Bevölkerung anhand empirischer Daten beobachten, beschreiben und analysieren (Noll 2013: 818; Pohl/Ott 2019: 284). Durch diese spezifische Perspektive und normative Orientierung ergeben sich Anforderungen bezüglich der Auswahl, Aufbereitung und Präsentation von Informationen, wobei hier Kriterien wie inhaltliche Systematik, zeitliche Kontinuität, Anschaulichkeit und Verständlichkeit eine wesentliche Rolle spielen (Noll 2013: 818; Pohl/Ott 2019: 284).

Ein zentrales Element in der Quantifizierung des Sozialraums bildet die Auswahl der Indikatoren (im Sinne von Anzeigern für Sachverhalte), anhand derer das latente Konstrukt Sozialraum anschaulich gemacht werden soll. Dabei steht man bei der Durchführung einer Sozialraumanalyse vor der Herausforderung, dass viele Sachverhalte (z. B. Benachteiligung) nicht direkt bzw. umfassend messbar sind (de Lange/Nipper 2018: 300; Burzan 2019: 1415). In einem Spannungsfeld zwischen Datenverfügbarkeit, Validität und räumlicher Genauigkeit muss ein Mittelweg zur indirekten Konstituierung solcher Phänomene gefunden werden (Hradil 1987: 62; Burzan 2019: 1419). So wäre es beispielsweise diskutabel, ob ein Indikator, wie Nicht-Besitz eines TV-Gerätes (oder privaten Pkw), als Proxy für die Messung von Armut dienen könnte, da hier nicht nur die individuelle Finanzkraft, sondern auch Wertzuschreibungen zum Tragen kommen (Burzan 2019: 1419).

Nebst der Auswahl muss auch über die Anzahl der verwendeten Indikatoren entschieden werden, wobei in gesamtgesellschaftlichen Abbildungen zumeist mehr Indikatoren verwendet werden als in dezidiert problemorientierten Ansätzen, wie z. B. beim Fokus auf die Armut oder Lebenssituation von Jugendlichen (Dohnke 2012: 148). Bei der Auswahl der Indikatoren kann man im deutschsprachigen Raum auf diverse Register- bzw. Survey-basierte Daten zurückgreifen, wobei beide Arten Implikationen mit sich bringen (Henckel/Kramer 2019: 18). Am einfachsten zur Verfügung stehen meist umfassende und regelmäßig erhobene administrative Daten der Sozialstatistik, wie z. B. Bevöl- 
kerungs- und Erwerbsstatistiken (Noll 2013: 817). Surveybasierte Daten, sofern sie nicht von der amtlichen Statistik, wie etwa dem Mikrozensus in Österreich ${ }^{5}$, regelmäßig erhoben werden, stehen oft nur punktuell zur Verfügung und weisen meist geringe Fallzahlen (Sample) auf, die keine statistischen Aussagen auf kleinräumiger Ebene zulassen. Ein Vorteil von Umfragedaten gegenüber der amtlichen Sozialstatistik (vor allem Registerdaten) liegt darin, dass die

${ }^{5}$ Vgl. https://www.statistik.at/web_de/frageboegen/private_ haushalte/mikrozensus/index.html (13.08.2021). abgebildeten Thematiken entlang spezifischer Fragestellungen entwickelt werden und Daten somit passgenauer, aber auch kostenintensiver, erhoben werden können (Hartmann/ Lengerer 2019: 1225).

In der Stadtplanung wären möglichst kleinräumige Sozialraumanalysen das Ideal, um für einzelne Gebiete passgenaue und differenzierte Handlungsoptionen entwickeln $\mathrm{zu}$ können. Leider wird die Sozialstatistik diesem Ideal vielfach nicht gerecht, da mit der steigenden geographischen Auflösung, wie z. B. Zählsprengel oder Baublöcke, oft die Verfügbarkeit von validen Daten sinkt, da diese entweder nicht kleinräumig erhoben wurden, die Fallzahlen zu ge-

Tabelle 1 Gegenüberstellung der Stärken und Schwächen in Rangreihen- bzw. Rangfolgeverfahren, Indexbildung, Faktorenanalyse/ Hauptkomponentenanalyse und Clusteranalyse

\begin{tabular}{|c|c|}
\hline Verfahren & Vorgehen \\
\hline $\begin{array}{l}\text { Rangrei- } \\
\text { hen- } \\
\text { bzw. } \\
\text { Rang- } \\
\text { folge- } \\
\text { verfah- } \\
\text { ren }\end{array}$ & $\begin{array}{l}\text { - Summarische Verfahren } \\
\text { - Rangfolgeverfahren führen Einzelkriterien in ein } \\
\text { Gesamtmaß zusammen, bevor dieses gereiht } \\
\text { wird } \\
\text { - Rangreihenverfahren reihen Indikatoren und } \\
\text { addieren die ordinalen Rangziffern }\end{array}$ \\
\hline $\begin{array}{l}\text { Index- } \\
\text { bildung }\end{array}$ & $\begin{array}{l}\text { - Primär modellgetriebenes multivariates statisti- } \\
\text { sches Verfahren } \\
\text { - Festgelegte Rechenvorschrift zur Zusammen- } \\
\text { führung robuster (Datenqualität), manifester } \\
\text { - meist metrischer - (Proxy-)Variablen zu einer } \\
\text { Maßzahl zur indirekten Konstituierung des Ana- } \\
\text { lyseobjekts }\end{array}$ \\
\hline $\begin{array}{l}\text { Faktoren-I } \\
\text { Haupt- } \\
\text { kompo- } \\
\text { nenten- } \\
\text { analyse }\end{array}$ & $\begin{array}{l}\text { - Primär datengetriebenes multivariates statisti- } \\
\text { sches Verfahren } \\
\text { - Manifeste Variablen werden mittels Korrelatio- } \\
\text { nen zu (Super-)Faktoren reduziert, welche nach } \\
\text { innen homogen, aber untereinander unabhängig } \\
\text { sind („Dimensionsreduktion“) } \\
\text { - Faktoren drücken die Datenvariabilität aus } \\
\text { - Die gebräuchlichste Form der Faktorenanalyse } \\
\text { stellt die Hauptkomponentenanalyse dar }\end{array}$ \\
\hline
\end{tabular}

Cluster- - Exploratives und strukturentdeckendes multivaanalyse riates statistisches Verfahren

- Gruppierung großer Datenmengen in Datenbündel (Cluster)

- Identifikation von Mustern („Dimensionsreduktion")

- Cluster nach innen homogen und nach außen heterogen. Ähnlichkeit über (euklidische) Distanz bemessen

- Es gibt hierarchische (z. B. Ward) und nichthierarchische (z. B. k-means) Clusteranalysen (Kombination empfohlen)

- Wahl des Clusteralgorithmus von Datenstruktur und Skalenniveau abhängig

\begin{tabular}{ll} 
Vorteile & Nachteile \\
\hline - Methodisch (sehr) ein- & - Kleine Änderungen in Inputdaten \\
fach & können große Auswirkungen auf die \\
- Können Grundlage für & Rangreihung haben \\
(un-)gewichtete additive & - Steigende Anzahl und Diversität der \\
Indexwerte bilden & Untersuchungsobjekte erschwert \\
& Rangbildung \\
& Ergebnisinterpretation \\
- Methodisch einfach & - Anfällig für Subjektivität bei der Fest- \\
(Rechenvorschrift) & legung der Indikatoren, Rechenvor- \\
- Theoriegetriebener An- & schriften und Gewichtungen
\end{tabular}

satz, der im Idealfall auf einem Erklärungsmodell beruht

\section{- Datengetriebener An- satz \\ - Erhält den größtmögli- chen Anteil der Gesamt- variation innerhalb des Originaldatensatzes - Größte Faktorladungen entfallen auf jene In- dikatoren, welche die größte Streuung über die Beobachtungen ha-} ben

- Ermöglicht Identifikation von Mustern und Strukturen in den Daten - Leicht verständliche und interpretierbare Ergebnisse
- Indikatorauswahl wichtiger als konzeptionelle Überlegungen

- Sensibel für Modifikationen der Ursprungsdaten

- Sensibel für Ausreißer

- Sensibel für kleine Samples

- Korrelationen zeigen nicht den realen Einfluss der Indikatoren auf das zu messende Phänomen

- Beitrag von Indikatoren ohne Korrelationen unterrepräsentiert

- Komplexes Verfahren, schwierig zu interpretieren

- Kein einheitliches Verfahren

- Ergebnis beeinflusst vom gewählten Clusteralgorithmus

- Ergebnis ist rein deskriptiv

- Benötigt detaillierte Dokumentation zur Nachvollziehbarkeit

- Korrelierende Indikatoren können das Ergebnis verzerren

- Nicht zur Hypothesentestung geeignet

- Zeitreihenanalysen problematisch

Quelle: Eigene Zusammenstellung nach OECD (2008); Bacher/Pöge/Wenzig (2010); Krause-Traudes (2014); Armstrong (2018); Backhaus/ Erichson/Plinke et al. (2018); de Lange/Nipper (2018); Holtbrügge (2018) 
ring sind oder die Daten aus rechtlichen Gründen nur begrenzt zur Verfügung stehen. Je größer die räumliche Analyseebene gewählt wird, desto ungenauer wird wiederum die Darstellung der Bevölkerungsstruktur in den einzelnen Gebieten und die Gefahr einer Homogenisierung der Bevölkerungsgruppen innerhalb der Raumeinheiten steigt, wohingegen üblicherweise die Verfügbarkeit von Daten und somit die Auswahl geeigneter Indikatoren zunimmt. Die letztendliche Entscheidung muss zwischen den beiden Polen, Datenverfügbarkeit und räumliche Analyseebene bei der Indikatorauswahl, abgewogen werden.

\subsection{Gegenüberstellung methodischer Ansätze in Sozialraumanalysen}

Die Quantifizierung nicht direkt beobachtbarer (latenter) Phänomene, wie etwa Benachteiligung im Kontext sozialräumlicher Analysen, kann über verschiedene statistische Verfahren erfolgen, welche vor allem metrisch skalierte (manifeste) Variablen als Proxy zur indirekten Konstituierung des Untersuchungsgegenstandes heranziehen. Dabei wird gleichzeitig versucht, komplexe Sachverhalte auf ein verständliches und einfach interpretierbares $\mathrm{Ma}$ zu reduzieren (de Lange/Nipper 2018: 300). Die zur Anwendung kommenden methodischen Ansätze reichen von einer vergleichsweise simplen Bildung von Reihungen (z. B. Rangfolge- oder Rangreihenverfahren) oder zusammenführenden Maßzahlen (z. B. Indexbildung) über komplexere faktoranalytische Verfahren zur Darstellung von Konstrukten mittels Dimensionsreduktion (z. B. Faktorenanalyse, Hauptkomponentenanalyse). Dazu kommen vergleichsweise abstrakte Clusteranalysen, die Muster und Strukturen in den Daten aufzeigen können (Bacher/Pöge/Wenzig 2010; Armstrong 2018: 13; Backhaus/Erichson/Plinke et al. 2018: 435; de Lange/Nipper 2018: 354). Jedes der benannten Verfahren ist für unterschiedliche Datenstrukturen und Fragestellungen anwendbar, wobei es vor einer Anwendung in einer Sozialraumanalyse Sinn hat, sich die jeweiligen Stärken und Schwächen der methodischen Ansätze zu vergegenwärtigen. In Tabelle 1 werden die Vorgehensweisen der jeweiligen Verfahren beschrieben, bei gleichzeitiger Gegenüberstellung damit einhergehender Vor- und Nachteile.

\section{Methodische Herangehensweise}

\subsection{Datengrundlage}

Als Datengrundlage für den methodisch-konzeptionellen Vergleich verschiedener Sozialraumberichterstattungen dienen die Projektberichte 25 deutschsprachiger Städte, die anhand eines integrativen Sozialraummonitorings Daten auf kleinräumiger Ebene systematisch auswerten. Mittels Internetrecherche wurde ersichtlich, dass nahezu jede Stadt(region) im deutschsprachigen Raum statistische Daten online bereitstellt, diese aber in unterschiedlichem Umfang aufbereitet werden (Markus 2009: 175).

Die Auswahl der analysierten Berichte erfolgte - angelehnt an Dohnke (2012: 144) - entlang der folgenden Kriterien: ${ }^{6}$

- (Groß-)Städte im deutschsprachigen Raum (in Österreich und der deutschsprachigen Schweiz mit mehr als 100.000 Einwohner/-innen, in Deutschland mit mehr als 200.000 Einwohner/-innen)

- Aktuellste verfügbare Publikation mit Erscheinungsdatum nach 2010 (Ausnahme: Basel 1999, Nürnberg 2004 und Aachen 2009)

- Öffentlicher Auftraggeber

- Auswertung kleinräumiger Raumeinheiten

- Systematische Analyse von Indikatoren, welche Entwicklungen in ein Verhältnis zueinander und/oder zur Entwicklung in der Gesamtstadt stellen

Generell beschäftigen sich in den untersuchten Städten - mit wenigen Ausnahmen - die städtischen Ämter - zuständig für Stadtplanung, Statistik oder Soziales - mit der Durchführung quantitativer Sozialraumanalysen. Von 52 bezüglich ihrer Bevölkerungszahl in Frage kommenden Städten verblieben lediglich 25 Städte, die alle oben genannten Kriterien erfüllen.

\subsection{Analytische Herangehensweise}

Die Studie orientiert sich am Vorgehen der qualitativen Metaanalyse (Schnepf/Groeben 2019). Dies ermöglicht, die Berichte sowohl hinsichtlich qualitativer Inhalte, wie die in den Berichten formulierte zugrunde liegende Motivation, als auch hinsichtlich quantitativer Daten der Sozialraumanalysen systematisch zu erforschen. Die Auswertung erfolgte dementsprechend in zwei Schritten: Erstens wurden zur allgemeinen übersichtlichen Texterfassung der Berichte in einer deduktiven Herangehensweise Informationen entlang der folgenden 19 Kategorien erfasst: Land, Stadt, Bezeichnung, Autorinnen/Autoren, Durchführende Institution(en), Jahr der Veröffentlichung, Datenstand, Zitiervorschlag, Link, Turnus, Datenbasis, Inhaltlicher Aufbau des

\footnotetext{
${ }^{6}$ Diese Studie stellt keinen Anspruch auf vollständige Erfassung aller - auf unsere Kriterien passenden - städtischen Bemühungen im deutschsprachigen Raum dar, da es womöglich auch nicht publizierte bzw. nach unserer Erhebung veröffentlichte Berichte geben kann (Stand: September 2020).
} 
Tabelle 2 Kategoriensystem zur induktiven Kodierung der Indikatoren

\begin{tabular}{|c|c|c|}
\hline Kategorie & Definition & Beispiel \\
\hline Bevölkerung & $\begin{array}{l}\text { Gesamtbevölkerung einer Stadt, ohne } \\
\text { Differenzierung in Bevölkerungsteile }\end{array}$ & Bevölkerungsdichte \\
\hline Alter & $\begin{array}{l}\text { Indikatoren zu spezifischen Alters- } \\
\text { gruppen }\end{array}$ & Anteil der Personen unter 18 Jahren an der Gesamtbevölkerung \\
\hline Geschlecht & Geschlechtsspezifische Indikatoren & Anteil der Frauen an der Gesamtbevölkerung \\
\hline $\begin{array}{l}\text { Haushaltszusam- } \\
\text { mensetzung }\end{array}$ & Bezug zu Haushaltsformen & Anteil der Einpersonenhaushalte an allen Haushalten \\
\hline Herkunft & $\begin{array}{l}\text { Indikatoren zur Herkunft von Bevöl- } \\
\text { kerungsteilen }\end{array}$ & $\begin{array}{l}\text { Anteil der Personen mit Migrationshintergrund an der Gesamtbevölke- } \\
\text { rung }\end{array}$ \\
\hline Bildung & $\begin{array}{l}\text { Indikatoren zu Bildungsstruktur bzw. } \\
\text { Bildungschancen }\end{array}$ & Anteile der Personen mit Hochschulreife an der Gesamtbevölkerung \\
\hline Sozialhilfe & Staatliche Transferleistungen & $\begin{array}{l}\text { Anteil der Leistungsempfänger/-innen nach SGB II an der Gesamtbevöl- } \\
\text { kerung }\end{array}$ \\
\hline $\begin{array}{l}\text { Einkommen/ Be- } \\
\text { schäftigung }\end{array}$ & $\begin{array}{l}\text { Indikatoren zur Einkommens- bzw. } \\
\text { Beschäftigungsstruktur }\end{array}$ & Median des steuerbaren Äquivalenzeinkommens der Privathaushalte \\
\hline $\begin{array}{l}\text { Hilfen zur Erzie- } \\
\text { hung }\end{array}$ & $\begin{array}{l}\text { Leistungen der Kinder- und Jugend- } \\
\text { hilfe für Familien mit Kindern }\end{array}$ & $\begin{array}{l}\text { Quote der Erziehungshilfemaßnahmen bezogen auf alle jungen Men- } \\
\text { schen im Alter von } 0 \text { bis } 20 \text { Jahren }\end{array}$ \\
\hline Mobilität & $\begin{array}{l}\text { Fluktuation inner- und außerhalb der } \\
\text { Stadtgrenzen }\end{array}$ & Wanderungen über Stadtgrenzen hinaus \\
\hline Wohnen & Bezug zur Wohnsituation & $\begin{array}{l}\text { Anteil der öffentlich inserierten Mietangebote im unteren Preisdrittel } \\
\left(2015 / 2016 \text { bis } 9,00 \text { Euro je } \mathrm{m}^{2}\right) \text { an allen öffentlich inserierten Mietan- } \\
\text { geboten }\end{array}$ \\
\hline Versorgung & $\begin{array}{l}\text { Nahversorgung, Infrastrukturen in- } \\
\text { nerhalb der untersuchten Raumein- } \\
\text { heiten }\end{array}$ & $\begin{array}{l}\text { Anteil der Personen, die zu Fuß (Dmax }=600 \mathrm{~m} \text { ) keinen Lebensmittel- } \\
\text { markt mit einer Mindestfläche von } 300 \mathrm{~m}^{2} \text { erreichen }\end{array}$ \\
\hline $\begin{array}{l}\text { Lebensqualität/ } \\
\text { Zufriedenheit }\end{array}$ & $\begin{array}{l}\text { Indikatoren zur Lebensqualität sowie } \\
\text { Zufriedenheit }\end{array}$ & Qualitative Bewertung der Wohnlage \\
\hline Sicherheit & $\begin{array}{l}\text { Indikatoren zur Sicherheit bzw. sub- } \\
\text { jektives Sicherheitsempfinden }\end{array}$ & Belastungsintensität Tatverdächtige \\
\hline Gesundheit & Gesundheitsspezifische Indikatoren & Anteil der übergewichtigen Kinder bei Schulbeginn an allen Kindern \\
\hline Partizipation & $\begin{array}{l}\text { Wahlbeteiligung, Bürgerschaftliches } \\
\text { Engagement }\end{array}$ & Wahlbeteiligung an der Kommunalwahl \\
\hline Religion & Religionsspezifische Indikatoren & Anteil der Evangelisch-Reformierten an der Gesamtbevölkerung \\
\hline
\end{tabular}

Berichts, Räumliche Analyseebene, Ziel der Sozialraumanalyse, Methodik, Oberkategorien, Indikatoren, Ausblick und Besonderes.

Zweitens wurde eine systematische Metaanalyse der in den einzelnen Sozialraumberichterstattungen verwendeten Indikatoren vorgenommen, indem diese zunächst separat für jeden Bericht induktiv gruppiert, anschließend - angeleitet durch die Dimensionen des Lebenslagenkonzepts systematisch in einem Analyseraster zusammengefasst und letztlich in Haupt- und Subdimensionen (vgl. Kapitel 4.2) kategorisiert wurden. Es wurden ausschließlich jene Indikatoren erfasst, welche Teil einer zusammenfassenden Analyse mittels multivariater Methodik waren. ${ }^{7}$ Im Rahmen einer

7 Zusätzliche Indikatoren, die nicht in die eigentliche Sozialraumanalyse eingeflossen sind, wurden in dieser Metaanalyse nicht berücksichtigt. induktiven Kodierung der verwendeten Indikatoren wurde das in Tabelle 2 angeführte System erstellt.

\section{Auswertungsergebnisse}

\subsection{Herausgeber, Motivationen und Ziele der Sozialberichterstattung}

Die Herausgeber der veröffentlichten Dokumente waren ausnahmslos die Kommunen selbst. Innerhalb der Verwaltung waren unterschiedliche Abteilungen mit der Bearbeitung befasst, z. B. Stadtentwicklung, Stadtplanung und/ oder Statistik (Stadt Wien 2013; Stadt Wien 2014; Landeshauptsstadt München 2016; Senatsverwaltung für Stadtentwicklung und Wohnen Berlin 2017; Stadt Wien 2017; Stadt Bern 2018; Stadt Köln 2018; Freie und Hansestadt Hamburg 2019; Senatsverwaltung für Stadtentwicklung und 
Wohnen Berlin 2019; Stadt Winterthur 2019), Sozialplanung (Stadt Frankfurt 2017a; Stadt Frankfurt 2017b; Landeshauptstadt Stuttgart 2018; Landeshauptstadt Hannover 2018), oder unterschiedliche Abteilungen in kooperativer Arbeit (Stadt Mannheim 2018; Stadt Münster 2019). Die Untersuchungen wurden entweder von den zuständigen Verwaltungseinheiten selbst vorgenommen (Senatsverwaltung für Stadtentwicklung und Wohnen Berlin 2017; Senatsverwaltung für Stadtentwicklung und Wohnen Berlin 2019; Stadt Winterthur 2019), wissenschaftlich begleitet (Stadt Wien 2013; Stadt Wien 2014; Stadt Wien 2017; Freie und Hansestadt Hamburg 2019) oder vollständig durch beauftragte wissenschaftliche Einrichtungen durchgeführt (Statistisches Amt des Kantons Basel-Stadt 1999). Der Umfang der Berichterstattung variiert stark. Während die Sozialraumanalyse in einigen Berichten nur einen Teilaspekt des Gesamtberichts wiedergibt (Statistisches Amt des Kantons Basel-Stadt (1999); Landeshauptstadt Hannover 2018; Stadt Bochum 2018), ist in anderen Berichten eine Sozialraumanalyse der Kern der Berichterstattung (Stadt Wien 2013; Stadt Wien 2014; Stadt Wien 2017; Stadt Köln 2018; Stadt Winterthur 2019). Die Motivation und

Tabelle 3 Häufigkeit der von den Indikatoren abgedeckten Haupt- und Teildimensionen nach Vergleichsstädten (in Prozent) sowie die Gesamtzahl der abgedeckten Indikatoren und Dimensionen

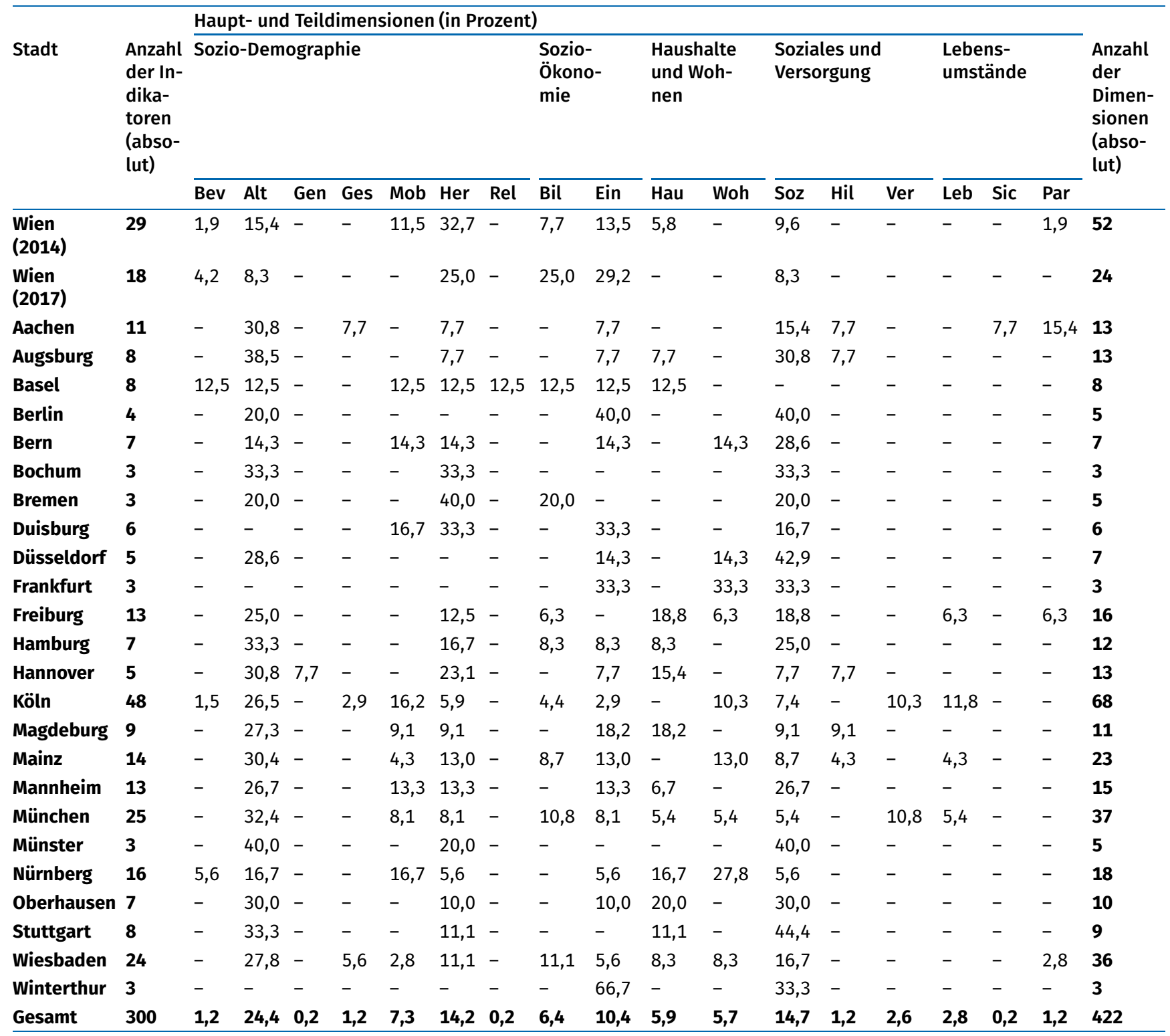

Teildimensionen: Bev = Bevölkerung, Alt = Alter, Gen = Geschlecht, Ges = Gesundheit, Mob = Mobilität, Fluktuation und Migration, Her $=$ Herkunft, Rel = Religion, Bil = Bildung, Ein = Einkommen und Beschäftigung, $\mathrm{Hau}=$ Haushalte, Woh $=$ Wohnen, Soz = Sozialhilfe, $\mathrm{Hil}=\mathrm{Hilfen}$ zur Erziehung, Ver = Versorgung, Leb = Lebensqualität und Zufriedenheit, Sic = Sicherheit, Par = Partizipation 
Zielsetzung(en) der Sozialraumanalysen wurden in allen untersuchten Berichten ähnlich formuliert und umfassen im Kern die folgenden Aspekte:

- Darstellung sozialräumlicher Unterschiede im Stadtgebiet (unter anderem Augsburg, Basel, Bern, Bochum, Duisburg, Hannover, Mannheim, Nürnberg, Wien)

- Sozialraumanalysen als Frühwarnsystem (Monitoring), um sozialräumliche Unterschiede zu identifizieren und gebietsspezifische Handlungsoptionen abzuleiten (unter anderem Berlin, Bremen, Düsseldorf, Frankfurt am Main, Freiburg, Hamburg, Magdeburg, Mainz, München, Münster, Wiesbaden, Winterthur).

- Schaffung eines Informations- und Planungsinstrumentes für Verwaltung, Politik, Fachkräfte der sozialen Arbeit und interessierte Öffentlichkeit (unter anderem Aachen, Köln, Oberhausen, Stuttgart, Wiesbaden)

Neben dem Umfang der Berichterstattungen unterscheiden sich auch die Zielgruppen, an die sich die Analysen rich- ten: Während einige Monitoringsysteme ausschließlich für verwaltungsinterne Zwecke entwickelt und die Ergebnisse nicht veröffentlicht werden (Stadt Wien 2013; Stadt Wien 2014; Stadt Wien 2017; Stadt Köln 2018; Stadt Winterthur 2019), stellen andere Kommunen der Öffentlichkeit sowohl die Ergebnisse der Sozialraumanalyse als auch weitere kontextualisierte Informationen zu Schwerpunktthemen zur Verfügung (Stadt Bochum 2018).

\subsection{Dimensionen und Indikatoren}

Insgesamt konnten für die 25 untersuchten Städte 300 Indikatoren dokumentiert werden, welche 422 Subdimensionen umfassen. Dabei ist die Anzahl der verwendeten Indikatoren in den einzelnen Berichterstattungen sehr unterschiedlich und variiert zwischen drei (Freie Hansestadt Bremen 2013; Stadt Frankfurt 2017a; Stadt Frankfurt 2017b; Stadt Bochum 2018; Stadt Münster 2019; Stadt Winterthur 2019) und 48 Indikatoren (Stadt Köln 2018). Im Wiener Kontext waren es beim Sozialraumatlas I 29 (Stadt Wien

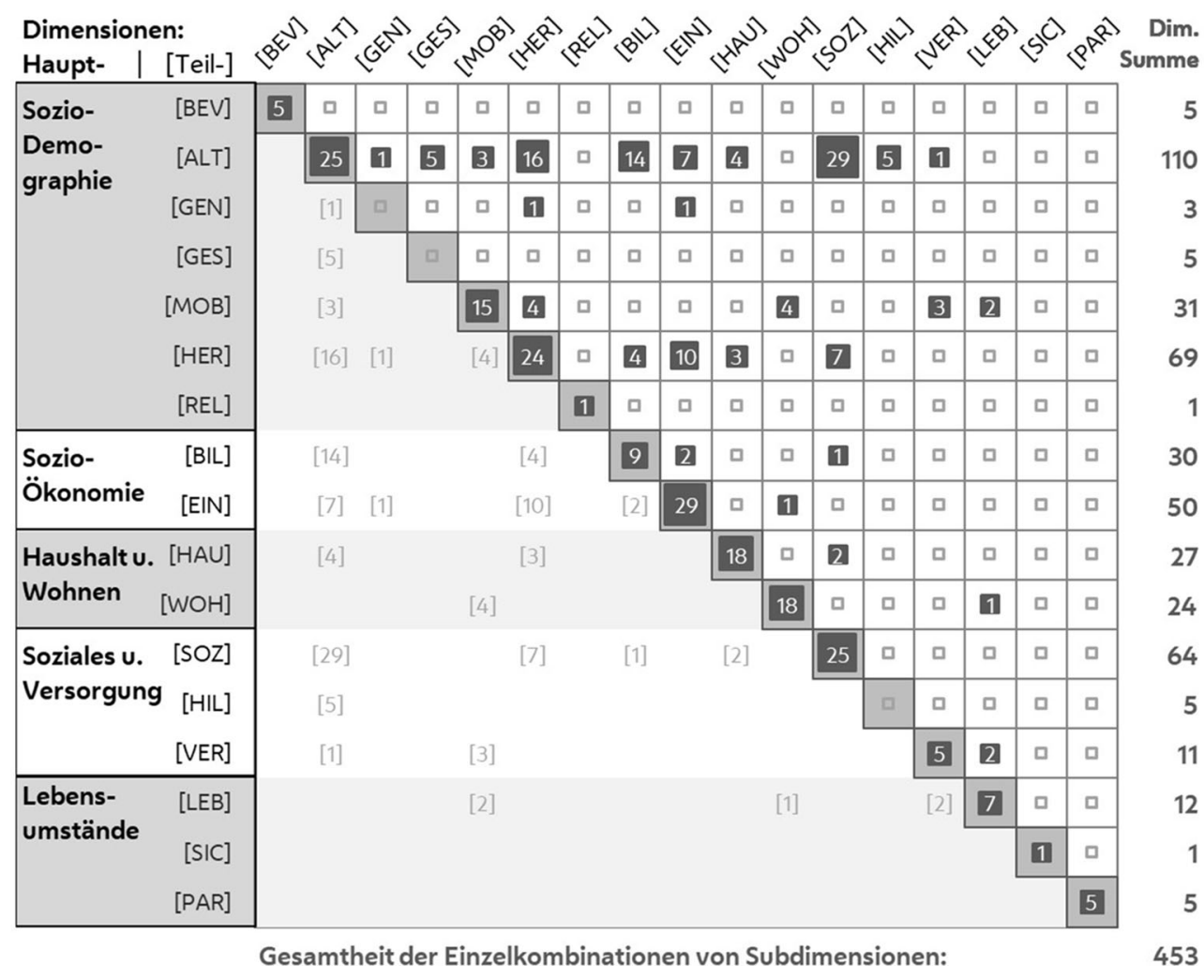

Abbildung 2 Häufigkeit der von den Indikatoren abgedeckten Haupt- und Teildimensionen nach Art des Auftretens (einzeln oder in Kombination)

Teildimensionen: Bev = Bevölkerung, Alt $=$ Alter, Gen $=$ Geschlecht, Ges $=$ Gesundheit, Mob = Mobilität, Fluktuation und Migration, Her = Herkunft, Rel = Religion, Bil = Bildung, Ein = Einkommen und Beschäftigung, Hau = Haushalte, Woh = Wohnen, Soz = Sozialhilfe, Hil = Hilfen zur Erziehung, Ver = Versorgung, Leb $=$ Lebensqualität und Zufriedenheit, Sic = Sicherheit, Par = Partizipation 
2013; Stadt Wien 2014) und bei dessen Neuauflage 18 Indikatoren (Stadt Wien 2017), welche in die Analyse einflossen. Im Rahmen der vorliegenden Untersuchung wurde eine quantitative Analyse der über alle Vergleichsstädte verwendeten Indikatoren durchgeführt, um daraus abzuleiten, welchen Themenfeldern bzw. Dimensionen besondere Bedeutung zukommt, wobei wir fünf Haupt- und 17 Teildimensionen unterscheiden (vgl. Tabelle 3).

Die einzelnen Indikatoren wurden je nach Merkmalsausprägung einer oder mehreren Haupt- und Teildimensionen zugeordnet. Wenn man zum Beispiel einen Indikator wie Jugendarbeitslosigkeit ${ }^{8}$ heranzieht (vgl. Landeshauptstadt Mainz 2018), kann dieser Indikator den Teildimensionen Alter sowie Einkommen und Beschäftigung zugewiesen werden. Damit ist dieser Indikator eine Kombination von zwei Teildimensionen. Wenn man die abgedeckten Teildimensionen nach ihrer Zuordnung zu den fünf Hauptdimensionen gruppiert, wird deutlich, dass der Bereich Sozio-Demographie und dessen sieben Teildimensionen rund 206-mal aufscheinen, gefolgt von Soziales und Versorgung (78), Sozio-Ökonomie (71), Haushalt und Wohnen (49) und Lebensumständen (18). Dabei ist es vor allem die Teildimension Alter (103), die deutlich vor Sozialhilfe (62) und Herkunft (60) zu den bestimmenden Dimensionen gehört. Themen wie Sicherheit (1), Religion (1) oder Geschlecht (3) sind vergleichsweise unterrepräsentiert bzw. werden über andere Studien und Publikationen abgedeckt, wie z. B. Lebensqualität und Zufriedenheit (12) (Verwiebe/Haindorfer/ Dorner et al. 2020). ${ }^{9}$

Nebst der reinen Erfassung der Frequenz einzelner Teildimensionen in den dokumentierten Indikatoren sind auch die auftretenden Merkmalkombinationen von Interesse. Dass Alter eine häufige Dimension im Sozialraummonitoring ist, hängt damit zusammen, dass es oft in Kombination (84mal) mit einer oder mehreren anderen Dimensionen auftritt (z. B. Jugendarbeitslosigkeit) und nur selten als Einzelindikator (25). Am häufigsten wird Alter mit den Teildimensionen Sozialhilfe (29), Herkunft (16) und Bildung (14) kombiniert (vgl. Abbildung 2). Obwohl die Dimension Alter am häufigsten gezählt werden kann, tritt sie zumeist in Kombination mit anderen Attributen, zur Quantifizierung von sozialer Ungleichheit, auf. Jedoch zielen die verwendeten Indikatoren in ihrem Kern auf Fragestellungen zum Arbeitsmarkt, zu Sozialhilfen und Bildungschancen ab. Dennoch kann attestiert werden, dass Ausprägungen sozialer

\footnotetext{
${ }^{8}$ Anteil der Arbeitslosen unter 25 Jahren an der Summe der sozialversicherungspflichtig Beschäftigten unter 25 Jahren und den Arbeitslosen unter 25 Jahren (Landeshauptstadt Mainz 2018: 300).

9 Vgl. https://www.graz.at/cms/beitrag/10317056/7749787/

Ergebnisse_der_Lebensqualitaet_Befragung.html (13.08.2021).
}

Ungleichheit vielfach für spezifische Alterssegmente separat betrachtet werden (z. B. Jugendliche, Pensionisten), um diese Gruppen bei der Ausarbeitung von Handlungsoptionen zum Ausgleich sozialer Ungleichheiten spezifisch zu adressieren.

Insgesamt konnten 453 Kombinationen von Teildimensionen identifiziert werden, wobei jede (bilaterale) Kombination zwischen Teildimensionen einzeln gezählt wurde. Bei einem Indikator wie beispielsweise Frauen-Jugendarbeitslosigkeit lassen sich mit (A) Alter, (B) Einkommen und Beschäftigung und (C) Geschlecht drei Teildimensionen ausweisen. Hier ergeben sich drei Einzelkombinationen: A-B, B-C und A-C, die einzeln gezählt werden. Gleichzeitig treten andere soziodemographische Dimensionen, wie Geschlecht, Gesundheit oder Religion weniger in Erscheinung.

\subsection{Räumliche Analyseebenen}

Auf der Ebene der Raumeinheiten kann die Anzahl der verwendeten Gebietseinheiten je Stadt und die durchschnittliche Zahl der Bewohnerinnen und Bewohner der Gebiete sehr unterschiedlich sein. Um Verzerrungen zu vermeiden, filtern einige Städte solche Gebiete heraus, welche besonders geringe Einwohnerzahlen aufweisen oder aber durch sonstige besondere Charakteristika geprägt sind, wie z. B. Gewerbegebiete (Senatsverwaltung für Stadtentwicklung und Wohnen Berlin 2017; Stadt Bern 2018; Freie und Hansestadt Hamburg 2019; Senatsverwaltung für Stadtentwicklung und Wohnen Berlin 2019). Die Ebene der gewählten Raumeinheit kann zwischen der kleinräumigen Baublockebene und größeren administrativen Teilräumen wie Stadtteilen oder Stadtbezirken schwanken. Einige Städte haben unabhängig von administrativen Raumeinheiten eigens für die Sozial(raum)planung Gebietseinheiten definiert, z. B. Lebensweltlich orientierte Räume (LOR) (Senatsverwaltung für Stadtentwicklung und Wohnen Berlin 2017), Sozialräume (Landeshauptstadt Düsseldorf 2018) oder Sozialmonitoringbezirke (Stadt Augsburg 2019). Dabei variiert sowohl die Anzahl der analysierten Teilräume als auch der Bewohnerinnen und Bewohner pro Teilraum zwischen den Städten erheblich. Während in Wien rund 7000 Baublöcke analysiert wurden und bei einer Einwohnerzahl von aktuell rund 1,91 Mio. (Stand: 1. Januar 2020) durchschnittlich 273 Personen in einer Analyseeinheit wohnen, waren es in Basel-Stadt gerade einmal 21 städtische Wohnviertel mit rund 8200 Einwohner/-innen pro Raumeinheit (dazu kamen in der Analyse weitere 86 Landgemeinden aus Basel-Landschaft). Die insgesamt geringste Anzahl an Gebietseinheiten nutzt Augsburg mit 22 Sozialmonitoringbezirken, in denen durchschnittlich 13.415 Personen wohnen. 
Über alle in diesem Beitrag analysierten Sozialraumanalysen hinweg gibt es somit eine beachtliche Spannweite an (Einwohner/-innen pro) Raumeinheiten, die in die Analysen eingeflossen sind, wobei es im Median 86 Raumeinheiten sind (Durchschnitt: 430) bzw. rund 5616 Einwohner/-innen pro Raumeinheit (Durchschnitt: 5772). Die Wahl der geeigneten Anzahl an Raumeinheiten bzw. der erfassten Bevölkerung pro Raumeinheit sowie der Indikatoren selbst hängt somit von der eigentlichen Fragestellung, Motivation sowie Datenverfügbarkeit ab. Je höher die räumliche Auflösung wird, desto kleiner wird der Pool an verlässlichen bzw. für die Fragestellung interessanten Daten, die verwendet werden könnten. Ein Beispiel hierfür sind Daten zu Einkommen oder Wohnkosten, welche häufig nur auf der Ebene der Gesamtstadt oder auf der Ebene der Stadtbezirke oder Stadtteile verfügbar sind. Hier stößt man bei feineren räumlichen Gliederungen an Grenzen der Datenverfügbarkeit.

Andererseits kann es sein, dass Daten, die für eine Sozialraumanalyse interessant wären, gar nicht erhoben werden, wie z. B. in Österreich die Religionszugehörigkeit (Speringer/Bauer 2014: 158; Goujon/Jurasszovich/Potančoková 2017: 7) oder nur unvollständig vorhanden sind, wie z. B. Mietpreisinformationen. Die Verfügbarkeit an Daten kann zwischen den Städten variieren und hat direkte Auswirkung auf die methodische Herangehensweise.

\subsection{Methodische Ansätze}

Die untersuchten Monitoringsysteme nutzen unterschiedliche, der jeweiligen Zielsetzung angepasste Methoden und können in vier Gruppen untergliedert werden: Rangfolgebzw. Rangreihenverfahren, Indexverfahren, Faktoren- bzw. Hauptkomponentenanalyse und Clusteranalyse (vgl. Tabelle 1).

Diese Methoden finden großteils einzeln Verwendung (21-mal) und werden lediglich in vier Städten in Kombina- tion angewendet: Aachen, Duisburg, München und Wien; dabei wird am häufigsten auf Indexverfahren (14-mal) und Clusteranalysen (9-mal) zurückgegriffen (vgl. Abbildung 3). Faktorenanalysen bzw. Hauptkomponentenanalysen kommen fünfmal zum Einsatz, allerdings ausschließlich in Kombination oder als Voranalyse zu Indexverfahren (Stadt Duisburg 2010; Landeshauptstadt München 2016) bzw. Clusteranalysen (Stadt Aachen 2009; Stadt Wien 2013; Stadt Wien 2014; Stadt Wien 2017). Die Ergebnisse der Faktorenanalysen bzw. Hauptkomponentenanalysen werden zur Bewertung der Indikatoren- und Bereichsgewichtungen in der Indexbildung oder als Eingangsdaten in die Clusteranalysen genutzt. Die Rangfolge- bzw. Rangreihenverfahren als einfachster methodischer Ansatz wurden lediglich dreimal, in Bern, Frankfurt am Main und Magdeburg angewendet, was an der limitierten Interpretationsfähigkeit dieser Verfahren liegen dürfte.

Ähnlich verhält es sich bei Clusteranalysen, welche zwar sehr anschaulich sind, aber schwierig zu interpretieren, wenn es um das Zustandekommen der Zusammensetzung der Cluster geht. Darüber hinaus erlauben Clusteranalysen keine Vergleiche zwischen mehreren Zeitpunkten, da sich mit jedem Durchlauf die Clusterparameter ändern. Die Popularität von Indexverfahren lässt sich auf deren methodische Einfachheit (Rechenvorschrift) und Interpretierbarkeit zurückführen, welche für die Umsetzung und Kommunikation der Ergebnisse von Vorteil ist.

\section{Fazit}

Quantitative Sozialraumanalysen sind eine gängige Herangehensweise zur Herausarbeitung von innerstädtischen sozialen Disparitäten und Veränderungsprozessen entlang demographischer, sozioökonomischer, kultureller und anderer Merkmale. Dabei besteht ihr Nutzen in der Identifikati-

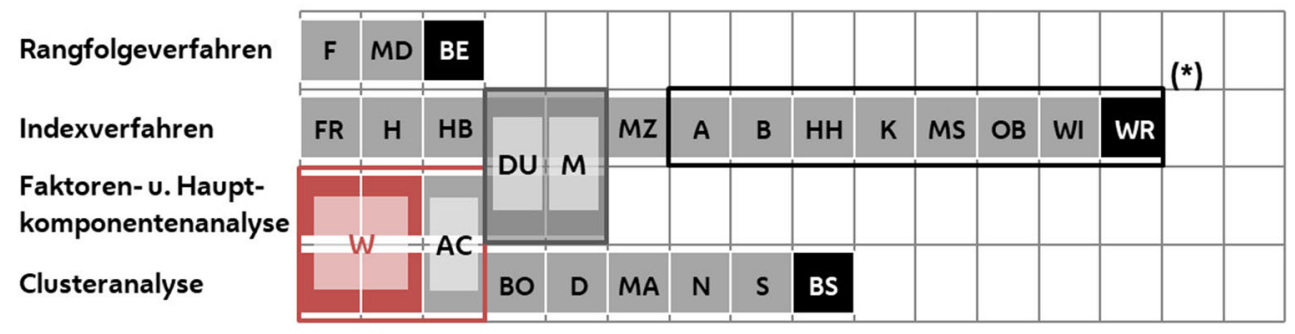

Abbildung 3 Überblick über die verwendeten methodischen Ansätze Notiz: Die Boxen beschreiben Kombinationen mehrerer methodischer Ansätze bzw. (*) Nutzung von Status- und Dynamikindex; Städtekürzel: Aachen (AC), Augsburg (A), Basel (BS), Berlin (B), Bern (BE), Bochum (BO), Bremen (HB), Duisburg (DU), Düsseldorf (D), Frankfurt (F), Freiburg (FR), Hamburg (HH), Hannover (H), Köln (K), Magdeburg (MD), Mainz (MZ), Mannheim (MA), München (M), Münster (MS), Nürnberg $(\mathrm{N})$, Oberhausen $(\mathrm{OB})$, Stuttgart $(\mathrm{S})$, Wien (W), Wiesbaden (WI), und Winterthur (WR) (in alphabetischer Reihenfolge). 
on potenzieller politischer Handlungsbedarfe sowie in der Anleitung von (stadt-)planerischen Maßnahmen. Die hier vorgestellte systematische komparative Metaanalyse sozialräumlicher Monitoringsysteme bzw. Sozialraumanalysen hat zum Ziel, verschiedene konzeptionelle und methodische Vorgehensweisen der 25 untersuchten Vergleichsstädte im deutschsprachigen Raum gegenüberzustellen, um daraus Erkenntnisse zu den unterschiedlichen Zielsetzungen und Motivationen, methodischen Herangehensweisen, analysierten Raumeinheiten sowie genutzten Merkmalen zur Darstellung der räumlichen Sozialstruktur zu gewinnen.

Die Ergebnisse zeigen, dass die gewählten Methodiken sowie die berücksichtigten thematischen Dimensionen stark von den stadtspezifischen Kontexten bzw. Zielsetzungen abhängen. Es gibt Städte, die einen engen thematischen Rahmen vorgeben und einen Fokus auf spezifische Dimensionen legen, wie Sozialversorgung (z. B. Stuttgart), Armut (z. B. Freiburg) oder (Jugend)arbeitslosigkeit (z. B. Düsseldorf), während andere gesamtgesellschaftliche Phänomene, wie soziale Ungleichheit (z. B. Berlin, München, Wien) oder Benachteiligung (z. B. Frankfurt am Main, Köln, Mainz), betrachten.

Dementsprechend gibt es eine breite Streuung an Dimensionen und Merkmalen, die unterschiedlich gehäuft Verwendung finden. Um eine quantitative Auswertung zu ermöglichen, wurde versucht, die in den einzelnen Sozialraumanalysen genutzten Indikatoren zu gruppieren und entlang der im Lebenslagenkonzept beschriebenen Dimensionen systematisch in ein Analyseraster zu übertragen. Hierbei konnte festgestellt werden, dass speziell die Teildimension Alter häufig (auch in Kombination) in den Analysen vorkommt, wenn es unter anderem um Kinder und Jugendliche in Kombination mit Sozialleistungen oder Beschäftigung geht. Eine andere Fokusgruppe bilden ältere Personen, welche für eine bedarfsgerechte Planung von sozialen Infrastrukturen bzw. die Gewährleistung von Sozialleistungen von hoher Bedeutung sind. Dies spiegelt sich in den dem Alter folgenden Dimensionen wider, namentlich Sozialhilfe, Herkunft sowie Einkommen und Beschäftigung.

Hier wäre es ratsam, die thematische Abdeckung ausgeglichener zu gestalten und andere Themenfelder stärker zu berücksichtigen, wenn man gesamtgesellschaftliche Strukturen und Prozesse aufzeigen will. Vielfach ist das allerdings nur eingeschränkt möglich, da man sich bei kleinräumigen Sozialraumanalysen immer im Spannungsfeld zwischen räumlicher Auflösung und Datenverfügbarkeit bewegen muss. Dadurch werden Themen oder ,weiche“ Indikatoren ohne flächendeckendes kleinräumiges Datenmaterial, wie z. B. Informationen zum subjektiven Sicherheitsempfinden oder Lebenszufriedenheit, wenig und gar nicht in quantitativen Sozialraumanalysen berücksichtigt. Somit können sich inhaltliche ,blinde Flecken ' herausbilden, die es bei der Interpretation zu bedenken gilt. Gleichzeitig kommen Themen, wie die zunehmenden gesellschaftlichen Differenzierungsprozesse (z. B. Pluralisierung von Werten) sowie beobachtbare soziale Segregation wohlhabender Teile der Bevölkerung (z. B. Gentrifizierung) selten in deutschsprachigen Sozialraumberichterstattungen vor (Dohnke/SeidelSchulze 2013; Pohl/Ott 2019: 299).

Die Limitation der Datenverfügbarkeit bedingt zudem, dass es bei den untersuchten Städten ein breites Spektrum an analysierten Raumeinheiten und Bewohnerinnen/ Bewohner in denselbigen gibt, welche von knapp $20 \mathrm{Be}-$ zirken oder Stadtteilen mit über 13.000 Einwohner/-innen bis zu über 7000 Baublöcken mit weniger als 300 Einwohner/-innen in Wien reichen, wobei der Median bei der Anzahl der räumlichen Einheiten unter 100 Raumeinheiten liegt. Um gehaltvolle Aussagen über die sozialräumlichen Disparitäten treffen zu können, gilt es, einen Mittelweg zwischen für die Fragestellung wichtigen Indikatoren und Dimensionen sowie der kleinräumigen Datenverfügbarkeit zur Festlegung der geeigneten räumlichen Auflösung zu finden.

Dies ist auch relevant für die Wahl der Methoden. Eine Mehrheit der untersuchten Städte griff auf Indexverfahren noch vor Clusteranalysen zurück. Die Hauptkomponentenbzw. Faktoranalysen dienten in den jeweiligen Forschungsdesigns aufgrund ihrer limitierten Interpretierbarkeit nur als Voranalyse und fanden alleinstehend keine Anwendung. Die Rangreihen- bzw. Rangfolgeverfahren fanden in den geringsten Fällen Anwendung, da sie trotz ihrer simplen Anwendung nur eine eingeschränkte Interpretationsfähigkeit besitzen. Prinzipiell wäre es ratsam, verschiedene methodische Ansätze zu testen und gegebenenfalls Methodenkombinationen zu verwenden.

Generell war festzustellen, dass lediglich knapp die Hälfte aller für die Analyse potenziell in Frage kommender Städte im deutschsprachigen Raum quantitative Sozialraumanalysen mit dem Versuch, Indikatoren und Dimensionen in eine Maßzahl zusammenzuführen, publiziert haben. Quantitative Sozialraumanalysen bergen durch ihre potenziell positivistische und kategoriale Darstellung der Gesellschaft die Gefahr einer Reproduzierung einer ,Schubladisierung “ von Stadtteilen, welche sich negativ auf deren öffentliches Image auswirken kann. Es ist anzunehmen, dass es Städte gibt, die derartige Studien durchgeführt haben, aber von einer Publikation absehen, um einer Stigmatisierung einzelner Stadtviertel vorzubeugen. Diese Analyse kann hier einen Anreiz liefern, dies reflexiv zu überdenken bzw. Bemühungen in diese Richtung zu initiieren und sich untereinander in Form von Städtekooperationen auszutauschen, um voneinander $\mathrm{zu}$ lernen.

Vieles hängt von der Zielsetzung bzw. den potenziellen Adressatinnen und Adressaten der Studien ab, z. B. 
städtische Akteure oder Verwaltungseinheiten. Hier wäre es ratsam, im Projektprozess frühzeitig eine Kooperation zwischen Fachbereichen (z. B. Stadtplanung, Statistik, Sozialplanung) anzuvisieren, um deren Anforderungen für die Verwertbarkeit solcher Analysen verstehen zu lernen und deren Expertise zu nutzen. Um in Planungsprozessen von Relevanz zu sein, sollten derartige Analysen auch eine Kontinuität aufweisen, das heißt in regelmäßigen Abständen (z. B. jährlich) durchgeführt werden, um innerstädtische Dynamiken verstehen zu lernen.

Die hier vorgestellte Metaanalyse stellt die unterschiedlichen konzeptionellen und methodischen Herangehensweisen bei quantitativen Sozialraumanalysen in 25 deutschsprachigen (Groß-)Städten systematisch gegenüber. Daraus lassen sich komparative Aussagen über die einer Sozialraumanalyse zugrunde liegenden Kernelemente, wie Motivationen, Ziele und inhaltlichen Fragestellungen, sowie die verwendeten Indikatoren, räumlichen Analyseebenen und methodischen Herangehensweisen der jeweiligen städtischen Analysen treffen. Der Erkenntnisgewinn kann für Stadt- und Regionalverwaltungen von Relevanz bei der (Neu-)Konzeption und Durchführung eigener Sozialraumanalysen sein. In einem weiteren Schritt wäre eine Erweiterung dieser Metaanalyse über den deutschsprachigen Raum hinaus denkbar, um Städte mit unterschiedlichen Stadtplanungstraditionen und mit unterschiedlichen demographischen, sozioökonomischen und politischen Rahmenbedingungen zu vergleichen.

\section{Literatur}

Armstrong, M. (2018): Armstrong's job evaluation handbook: a guide to achieving fairness and transparency in pay and reward. London.

Bacher, J.; Pöge, A.; Wenzig, K. (2010): Clusteranalyse: Anwendungsorientierte Einführung in Klassifikationsverfahren. München. https://doi.org/10.1524/ 9783486710236

Backhaus, K.; Erichson, B.; Plinke, W.; Weiber, R. (2018): Multivariate Analysemethoden: eine anwendungsorientierte Einführung. Berlin.

Bauer, R.; Haydn, G. (2019): Wiener Gemeindebauten. Von der Wohnungsnot im 19. Jahrhundert zum sozialen Wohnbau im Roten Wien. In: Stadt Wien (Hrsg.): Das Rote Wien in Zahlen: 1919-1934. Wien, 38-45.

BBSR - Bundesinstitut für Bau- Stadt- und Raumforschung (Hrsg.) (2019): Soziale Vielfalt im Blick. Stadtquartiere unter Nachfragedruck. Bonn. = BBSR-Online-Publikation $07 / 2019$.

Bourdieu, P. (1982): Die feinen Unterschiede: Kritik der gesellschaftlichen Urteilskraft. Frankfurt am Main.

Burzan, N. (2011): Soziale Ungleichheit: Eine Einführung in die zentralen Theorien. Wiesbaden. https://doi.org/10. 1007/978-3-531-93154-8

Burzan, N. (2019): Indikatoren. In: Baur, N.; Blasius, J. (Hrsg.): Handbuch Methoden der empirischen Sozialforschung. Wiesbaden, 1415-1422. https://doi.org/10.1007/ 978-3-658-21308-4_105

de Lange, N.; Nipper, J. (2018): Quantitative Methodik in der Geographie. Paderborn.

Dirksmeier, P. (2010): Super-Gentrification und metropolitaner Habitus: eine Kritik jüngerer Entwicklungen in der britischen Gentrificationforschung. In: Raumforschung und Raumordnung 68, 6, 447-457. https://doi.org/10. 1007/s13147-010-0065-z

Dohnke, J. (2012): Monitoringsysteme der sozialen Stadtentwicklung - Stand und zukünftige Herausforderungen. In: Pohlan, J.; Glasauer, H.; Hannemann, C.; Pott, A. (Hrsg.): Jahrbuch Stadtregion 2011/2012: Schwerpunkt Stadt und Religion. Opladen, 144-169. https://doi.org/ 10.3224/jbsr.v7i1.08

Döringer, S.; Uchiyama, Y.; Penker, M.; Kohsaka, R. (2020): A meta-analysis of shrinking cities in Europe and Japan. Towards an integrative research agenda. In: European Planning Studies 28, 9, 1693-1712. https:// doi.org/10.1080/09654313.2019.1604635

Dohnke, J.; Seidel-Schulze, A. (2013): Soziale Segregation und Polarisierung in deutschen Städten - Relevanz und Möglichkeiten der Messung. In: Information zur Raumentwicklung 6, 529-539.

Farwick, A. (2012): Segregation. In: Eckardt, F. (Hrsg.): Handbuch Stadtsoziologie. Wiesbaden, 381-419. https:// doi.org/10.1007/978-3-531-94112-7_18

Franz, Y. (2015): Gentrification in Neighbourhood Development. Case Studies from New York City, Berlin and Vienna. Göttingen.

Freie Hansestadt Bremen (2013): Monitoring Soziale Stadt Bremen 2013. Bremen.

Freie und Hansestadt Hamburg (2019): Sozialmonitoring Integrierte Stadtteilentwicklung. Ergebnisbericht. Hamburg.

Friedrichs, J. (2013): Sozialräumliche Kontexteffekte der Armut. In: Oberwittler, D.; Rabold, S.; Baier, D. (Hrsg.): Städtische Armutsquartiere - Kriminelle Lebenswelten? Wiesbaden, 11-44. https://doi.org/10.1007/978-3-53193244-6_1

Geißler, R. (2014): Facetten der modernen Sozialstruktur. In: Bundeszentrale für Politische Bildung (Hrsg.): Sozialer Wandel in Deutschland. Bonn, 74-83. = Informationen zur politischen Bildung 324.

Gennies, M. (2021): Konzeptverfahren als Instrument einer gemeinwohlorientierten Stadtentwicklung. Berlin. $=$ ISR Impulse Online 68. https://doi.org/10.14279/ depositonce-10398 
Glaeser, E. L.; Resseger, M.; Tobio, K. (2009): Inequality in Cities. In: Journal of Regional Science 49, 4, 617-646. https://doi.org/10.1111/j.1467-9787.2009.00627.x

Glatter, J.; Wiest, K. (2008): Gentrifizierungstendenzen unter den Bedingungen des Mietermarktes? Zum Wandel innenstadtnaher Quartiere in ostdeutschen Städten seit der Wiedervereinigung. In: Gestring, N.; Glasauer, H.; Hannemann, C.; Petrowsky, W.; Pohlan, J. (Hrsg.): Jahrbuch Stadt Region 2007/2008. Arme reiche Stadt. Opladen, 55-72.

Goujon, A.; Jurasszovich, S.; Potančoková, M. (2017): Religious Denominations in Vienna \& Austria: Baseline Study for 2016 - Scenarios until 2046. Wien.

Häußermann, H. (2010): Armutsbekämpfung durch Stadtplanung? In: Aus Politik und Zeitgeschichte 51-52, 23-29.

Häußermann, H.; Siebel, W. (2004): Stadtsoziologie: eine Einführung. Frankfurt am Main.

Hartmann, P. H.; Lengerer, A. (2019): Verwaltungsdaten und Daten der amtlichen Statistik. In: Baur, N.; Blasius, J. (Hrsg.): Handbuch Methoden der empirischen Sozialforschung. Wiesbaden, 1223-1231. https://doi.org/10. 1007/978-3-658-21308-4_89

Hartwig, J. (2009): Einführung: Den demografischen und sozialen Wandel steuern - Gründe für ein Sozialmonitoring auf kommunaler Ebene. In: Hartwig, J. (Hrsg.): Sozialmonitoring - Steuerung des demografischen und sozialen Wandels. Berlin, 7-38. = Planung und Organisation 5.

Heintel, M.; Speringer, M.; Schnelzer, J.; Bauer, R. (2017): Multipler Benachteiligungsindex. Fallbeispiel Oberpinzgau. In: Mitteilungen der Österreichischen Geographischen Gesellschaft 159, 173-198. https://doi.org/10. 1553/moegg159s173

Helbrecht, I. (2016): Gentrifizierung und Verdrängung. In: Helbrecht, I. (Hrsg.): Gentrifizierung in Berlin. Verdrängungsprozesse und Bleibestrategien. Bielefeld, 9-16.

Henckel, D.; Kramer, C. (2019): Zeitgerechte Stadt - Eine Einführung. In: Henckel, D.; Kramer, C. (Hrsg.): Zeitgerechte Stadt. Konzepte und Perspektiven für die Planungspraxis. Hannover, 5-22. = Forschungsberichte der ARL 9.

Heye, C.; Leuthold, H. (2004): Das Konzept des „,sozialgeographischen Raumes“. Theoriegeleitete Sozialraumanalyse unter den Bedingungen einer individualisierten Gesellschaft. Zürich.

Holtbrügge, D. (2018): Personalmanagement. Berlin.

Hradil, S. (1987): Sozialstrukturanalyse in einer fortgeschrittenen Gesellschaft: Von Klassen und Schichten zu Lagen und Milieus. Opladen.

Kadi, J.; Verlič, M. (Hrsg.) (2019): Gentrifizierung in Wien. Perspektiven aus Wissenschaft, Politik und Praxis. Wien.
Keeley, B. (2015): Income Inequality: The Gap between Rich and Poor. Paris. https://doi.org/10.1787/978926424 6010-en

Krause-Traudes, M. A. (2014): Raumbezogenes Monitoring als Aufgabe eines integrierten kommunalen Informationsmanagements. Konzept, Methodik und Grenzen. Dissertation an der Universität zu Köln.

Küpper, P.; Peters, J. C. (2019): Entwicklung regionaler Disparitäten hinsichtlich Wirtschaftskraft, sozialer Lage sowie Daseinsvorsorge und Infrastruktur in Deutschland und seinen ländlichen Räumen. Braunschweig. = Thünen-Report 66. https://doi.org/10.3220/ REP1547565802000

Küpper, P.; Steinführer, A. (2017): Daseinsvorsorge in ländlichen Räumen zwischen Ausdünnung und Erweiterung: ein Beitrag zur Peripherisierungsdebatte. In: Europa Regional 23, 4, 44-60.

Landeshauptstadt Düsseldorf (2018): Sozialräumliche Gliederung. Fortschreibung 2017. Düsseldorf. = Statistik und Stadtforschung 56.

Landeshauptstadt Hannover (2018): Sozialbericht 2018. Soziale Lagen, Stadtteile und Generationen. Hannover.

Landeshauptstadt Mainz (2018): Sozialraumanalyse Mainz 2017. Fortschreibung der Analyse der sozialräumlichen Struktur der Landeshauptstadt Mainz. Mainz.

Landeshauptstadt München (2016): Münchner Stadtteilstudie. Fortschreibung 2015. München.

Landeshauptstadt Stuttgart (2018): Sozialdatenatlas. Darstellung und Analyse der sozialen Situation in der Landeshauptstadt Stuttgart mit Daten aus dem Jahr 2016. Stuttgart.

Lawrence, R. J. (2002): Inequalities in Urban Areas: Innovative Approaches to Complex Issues. In: Scandinavian Journal of Public Health 30, 59 suppl, 34-40. https://doi. org/10.1177/14034948020300030601

Mardorf, S. (2006): Konzepte und Methoden von Sozialberichterstattung: eine empirische Analyse kommunaler Armuts- und Sozialberichte. Wiesbaden. https://doi.org/ 10.1007/978-3-531-90372-9

Markus, G. (2009): Kein Sozialmonitoring ohne Sozialindikatoren - eine Handlungsanleitung für den Aufbau von Sozialindikatoren. In: Hartwig, J. (Hrsg.): Sozialmonitoring - Steuerung des demografischen und sozialen Wandels. Berlin, 175-194. = Planung und Organisation 5.

Mau, S. (2018): Das metrische Wir. Über die Quantifzierung des Sozialen. Bonn.

Milanović, B. (2016): Global Inequality. A New Approach for the Age of Globalization. Cambridge.

Nijman, J.; Wei, Y. D. (2020): Urban Inequalities in the 21st Century Economy. In: Applied Geography 117, 102188. https://doi.org/10.1016/j.apgeog.2020.102188

Noll, H.-H. (2013): Sozialstatistik und Sozialberichter- 
stattung. In: Mau, S.; Schöneck, N.M. (Hrsg.): Handwörterbuch zur Gesellschaft Deutschlands. Wiesbaden, 816-829. https://doi.org/10.1007/978-3-531-18929-1_ 56

OECD - Organisation for Economic Co-operation and Development (2008): Handbook on constructing composite indicators: methodology and user guide. Paris.

OECD - Organisation for Economic Co-operation and Development (2018): Divided Cities: Understanding Intra-Urban Inequalities. Paris. https://doi.org/10.1787/ 9789264300385-en

Pohl, T.; Ott, T. (2019): Von der Sozialraumanalyse zum Sozialraummonitoring: Evolution eines quantitativen Raumanalyseverfahrens. In: Geographische Zeitschrift 107, 4, 282-304. https://doi.org/10.25162/gz-2019-0016

Schmidt, G. (2011): Urban Governance im Spannungsfeld zwischen Partizipation und Effektivität: Erfahrungen mit dem New Deal for Communities-Programm in Bristol. In: Frey, O.; Koch, F. (Hrsg.): Die Zukunft der Europäischen Stadt: Stadtpolitik, Stadtplanung und Stadtgesellschaft im Wandel. Wiesbaden, 229-245. https://doi.org/ 10.1007/978-3-531-92653-7_14

Schnelzer, J. (2018): Versteckte Gentrifizierung: Zum Verhältnis von Gentrifizierung, Habitus und Raum. Masterarbeit an der Universität Wien.

Schnepf, J.; Groeben, N. (2019): Qualitative Metaanalyse mithilfe computergestützter qualitativer Inhaltsanalyse am Beispiel von Lokale-Agenda-21-Prozessen. In: Forum Qualitative Sozialforschung 20, 3, 1-20. https://doi. org/10.17169/fqs-20.3.3313

Schulte-Haller, M. (2011): Soziale Mischung und Quartierentwicklung: Anspruch versus Machbarkeit. Bern.

Senatsverwaltung für Stadtentwicklung und Wohnen Berlin (2017): Monitoring Soziale Stadtentwicklung Berlin 2017. Berlin.

Senatsverwaltung für Stadtentwicklung und Wohnen Berlin (2019): Monitoring Soziale Stadtentwicklung Berlin 2019. Berlin.

Shevky, E.; Bell, W. (1961): Social Area Analysis. In: Theodorson, G. (Hrsg.): Studies in Human Ecology. New York, 226-235.

Siebel, W. (2012): Die europäische Stadt. In: Eckardt, F. (Hrsg.): Handbuch Stadtsoziologie. Wiesbaden, 201-211. https://doi.org/10.1007/978-3-531-94112-7_10

Speringer, M.; Bauer, R. (2014): Residential Patterns by Religion and Ethnicity in Vienna. In: Grim, B.; Johnson, T.; Skirbekk, V.; Zurlo, G. (Hrsg.): Yearbook of International Religious Demography 2014. Leiden, 157-166.

Speringer, M.; Schnelzer, J.; Heintel, M. (2020): Quantifizierung regionaler sozioökonomischer Disparitäten in ländlichen Regionen. Am Beispiel der Leaderregion Südliches Waldviertel-Nibelungengau, Österreich. In: Europa Regional 26, 3, 55-72.

Stadt Aachen (2009): Sozialentwicklungsplan Aachen. Teil 1: Bericht zur sozialen Lage in Aachen. Aachen.

Stadt Augsburg (2019): Sozialmonitoring 2019. Daten zur Sozialberichterstattung. Augsburg.

Stadt Bern (2018): Monitoring Sozialräumliche Stadtentwicklung 2017. Bern.

Stadt Bochum (2018): Sozialbericht Bochum 2018. Bochum.

Stadt Duisburg (Hrsg.) (2010): Kleinräumiges Sozialraummonitoring in Duisburg. Duisburg. = Duisburger Kurzbeiträge zur Statistik und Stadtforschung, trendInfo 08/2010.

Stadt Frankfurt (2017a): Monitoring 2017. Zur sozialen Segregation und Benachteiligung in Frankfurt am Main. Teil 1. Frankfurt am Main. = Materialienreihe Jugend und Soziales 8.1.

Stadt Frankfurt (2017b): Monitoring 2017. Zur sozialen Segregation und Benachteiligung in Frankfurt am Main. Teil 2. = Materialienreihe Jugend und Soziales 8.2.

Stadt Köln (2018): Monitoring Stadtentwicklung Köln 2018. Köln.

Stadt Mannheim (2018): Mannheimer Sozialatlas 2017. Bevölkerung und soziale Lebenslagen. Mannheim.

Stadt Münster (2019): Sozialmonitoring 2018. Berechnungen, Tabellen und Karten. Münster.

Stadt Wien (2013): Sozialraumatlas Wien I. ZSI - Technischer Bericht (unveröffentlichtes Manuskript). Wien.

Stadt Wien (2014): Sozialraumatlas Wien I. ZSI - Projektbericht (unveröffentlichtes Manuskript). Wien.

Stadt Wien (2017): Sozialraumatlas Wien II. MA 18 - Projektbericht (unveröffentlichtes Manuskript). Wien.

Stadt Winterthur (2019): Sozialmonitoring als Instrument zur Koordination von Sozialarbeit und Stadtentwicklung. Winterthur.

Statistisches Amt des Kantons Basel-Stadt (Hrsg.) (1999): Bevölkerungsstruktur und Bevölkerungsdynamik beider Basel. Basel. = Stadt und Region 1.

Verwiebe, R.; Haindorfer, R.; Dorner, J.; Liedl, B.; Riederer, B. (2020): Lebensqualität in einer wachsenden Stadt. Endbericht. Wiener Lebensqualitätsstudie 2018 (Sozialwissenschaftliche Grundlagenforschung III). Wien.

Wunderlich, H. (2019): Kommunale Rahmenbedingungen und Herausforderungen für die Sozialpolitik und die Soziale Arbeit - Gemeindetypisierung für das Land Niedersachsen mit den Daten des ,Wegweiser Kommune'. https://www.holger-wunderlich.de/downloads/ (12.08.2021). 